\title{
Conditions for the successful integration of Human and Organizational Factors (HOF) in the nuclear safety analysis
}

\author{
Michèle Tosello ${ }^{\mathrm{a},}$, Françoise Lévêque ${ }^{\mathrm{b}}$, Stéphanie Dutillieu ${ }^{\mathrm{a}}$, Guillaume Hernandez ${ }^{\mathrm{c}}$ and Jean-François \\ Vautier, ${ }^{\mathrm{c}, \mathrm{d}}$ \\ The authors are members of the Human and Organizational Factors network of the CEA (French Alternative \\ Energies and Atomic Energy Commission), France

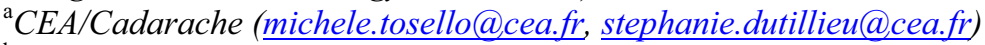 \\ ${ }^{\mathrm{b}} \mathrm{CEA}$ /Marcoule (francoise.leveque@cea.fr) \\ ${ }^{\mathrm{C} C E A / F o n t e n a y-a u x-R o s e s}$ (guillaume.hernandez@.cea.fr, jean-francois.vautier@.cea.fr) \\ ${ }^{\mathrm{d}}$ Professor assistant at the INSTN (National Institute for Nuclear Science and Technology), France
}

\begin{abstract}
This communication presents some elements which come from the experience feedback at CEA about the conditions for the successful integration of HOF in the nuclear safety analysis. To point out some of these conditions, one of the concepts proposed by Edgar Morin to describe the functioning of "complex" systems: the dialogical principle has been used. The idea is to look for some dialogical pairs. The elements of this kind of pair are both complementary and antagonist to one another. Three dialogical pairs are presented in this communication. The first two pairs are related to the organization of the HOF network and the last one is related to the methods which are used to analyse the working situations. The three pairs are: specialist - non-specialist actors of the network, centralized - distributed human resources in the network and microscopic - macroscopic levels of HOF methods to analyse the working situations. To continuously improve these three dialogical pairs, it is important to keep the differences which exist between the two elements of a pair and to find and maintain a balance between the two elements of the pairs.
\end{abstract}

Keywords: dialogical pairs, complex systems, human and organizational factors, nuclear safety

\section{Introduction}

This communication presents some elements which come from the experience feedback at CEA about the conditions for the successful integration of HOF in the nuclear safety analysis.

In other words the question is: what can be pointed out to explain a successful integration of HOF in this kind of analysis? A HOF network has been gradually developed over several years at CEA. Its development was presented previously [15]. Therefore, this communication is only focused on some structural conditions.

After reviewing the context of the CEA, the communication will present the principle used to point out some of the conditions set up to take HOF into account. Next, the application of this principle to the description of some characteristics of the HOF network will be presented. Finally, a discussion will be proposed to end this communication.

\section{Description of the CEA context}

CEA is a research organization which is dedicated to the development of research programs in relation to energy fields, in particular nuclear energy but also alternative energies. It consists of ten research centers located in different counties in France. Each CEA center has different facilities used to carry out its re-

*Corresponding author. E-mail: michele.tosello@cea.fr. 
searches. These nuclear facilities consist of "big tools", such as experimental reactors to conduct research on materials and research laboratories to perform studies on fuel. There are also service facilities which manage, treat and store waste which is provided by the experimentations. Each nuclear facility is managed by dedicated teams, including operators and experimenters. The working situations, which will be talked about later in this communication, are defined in this context, often qualified such as a context of risk activities.

Nuclear safety covers all the provisions to ensure the normal functioning of the nuclear facilities. It implies the prevention of accidents and the mitigation of their effects. The provisions consist of both the technical means and the organizational and human resources. Nuclear safety is a constant concern from the early design studies to the operational steps and lastly the dismantling.

CEA has both to set up the adapted provisions and to prove that the safety is ensured in the operational facilities. It has to carry out studies to demonstrate the conditions of safety. The human and organizational factors are involved in this kind of demonstration. Therefore, dedicated HOF studies which are focused on safety in working situations are carried out, and presented in the safety case, in relationship with the different steps of the facilities design.

The safety analysis was focused for a long time on technical points. The more recent integration of the $\mathrm{HOF}$ approach at CEA (for over ten years now) relies on a network made of dedicated competences.

\section{Principle used to analyze conditions for the successful integration of $\mathrm{HOF}$}

1. The authors have built a working group to take into account the experience feedback at CEA about the integration of HOF into the nuclear safety analysis. To point out some of these conditions for successful integration of $\mathrm{HOF}$, one of the concepts proposed by Edgar Morin $[8,9]$ to describe the functioning of "complex" systems: the dialogical principle has been used. The key point is to focus on dialogical pairs [2].

2. To briefly explain dialogical ways, the idea is to look for some pairs of elements which are both complementary and antagonist to one another. When using "antagonist" it means that the two elements of the pair work in opposite directions. Moreover, Edgar Morin indicates [4, 7] that in a dialogical way, the elements are also opposite, competing... one against the other. Therefore, in this communication, in order to be as simple as possible, a focus is made on the notion of complementarity between the antagonists and just one of the meanings of the word "opposite" is used. In this way, each element of the pair represents the antonym of the other.

3. For example, the brake and accelerator in a car are dedicated, respectively, to increasing and decreasing the velocity of the vehicle. But they are also complementary since with just one of them it is impossible to drive a car correctly. In the same way, we know that flexor and extensor muscles are necessary to walk and that with both a left hand and a right one we can hold a ball better than with just one big hand...

4. By the application of this concept, the conditions for the successful integration of HOF, which will be pointed out later in this paper, will be represented as pairs of elements.

5. Three dialogical pairs are presented in this communication. The first two pairs are related to the organization of the HOF network and the last one to the methods which are used to analyse the working situations:

- specialist - non-specialist actors of the network,

- centralized - distributed human resources in the network,

- microscopic - macroscopic levels of HOF methods to analyse the working situations.

\section{Analysis of the conditions of HOF integration into the safety analysis with the application of the "dialogical" concept}

When examining these three dialogical pairs, here are some of the conditions for success which can appear.

\subsection{Specialist-non-specialist actors of the network}

The CEA HOF network has been gradually developed over ten years. One of the objectives of this increase has been to extend the HOF culture into the CEA facilities. While, at the beginning, it included only a few HOF specialists, currently it includes both 
HOF specialists and non-specialists (mostly safety engineers), all together in the CEA. Each of them has dedicated tasks to do and specific methods to use, according to their respective skills and the level of difficulty of the analysis to provide. The specialists have to carry out in depth analyses in contrast with the analyses of non-specialists which have only to point out some prominent elements. The first ones use the usual methods of HOF specialists to conduct the HOF studies (interviews, detailed examinations of the functioning of working situations - most based on observations,...) [11] and the second ones use checklists [1] and special guidelines, not to conduct studies, but rather to help them to focus their attention on the different factors of the working situations.

Therefore, even if the methods of analysis are different, these two kinds of actors are complementary and together they can cover different types of situations to be analyzed.

Indeed, carrying out an in depth analysis is required in some cases, in particular for the every ten years safety re-examination of the facilities, or some complex situations requiring an expert examination. Meanwhile, some lighter analyses can be provided thru the non-specialists contributions. For example, in some events the non-specialists identify some $\mathrm{HOF}$ which are involved, such as difficulties in relation to the technical means used by the humans, the organization of the team or the physical environmental conditions. They propose a "first level" analysis of the event.

This distribution of the tasks, in particular allowing the non-specialists to do some analyses, is a way to develop a general safety culture [3] in facilities.

Finally, the experience feedback of the functioning of the network shows that it is very important that HOF specialists and non-specialists work well together. On the one hand, a study of a specialist requires good preparation and follow-up from the nonspecialists who are "in the field" in the facilities. On the other hand a non-specialist analysis has to be accompanied and checked by a specialist.

To ensure a good understanding and efficient communication between the actors of the network, the complementarity must be based on shared values.

In this way, the basic concepts of HOF from the social sciences, in particular from an ergonomics standpoint (the discipline which is currently most significant in our field), are developed for all the actors of the HOF network. As such, the model of the "working situations" based on the main factors which influence human performance at work is one of the most significant concepts to share. Another example concerns the importance of having shared knowledge about the difference between an error and a violation [10]. Indeed, because of the consequences of these kinds of potential human failures in a high risk industry such as nuclear energy, it is necessary to go beyond the use of the word "error" and to be able to discuss the nuances of the concept and the conditions related to the production of the "error". In the same field, according to INSAG-4 [3], knowledge could be shared on the concepts of negligence or infringement, linked with specific measures to take.

So, in practice, the creation of the CEA HOF network including non-specialists was accompanied by two conditions: promoting and managing how specialists and non-specialists work together and share common knowledge about HOF in order to communicate efficiently. Therefore, on the one hand, a dedicated training on HOF area for HOF non-specialists, who are mostly technical or safety engineers, has been set up. On the other hand, the HOF specialists have to be trained in the basic and technical principles of nuclear safety, and also in the safety organization principles.

\subsection{Centralized-distributed human resources in the network}

Due to the organization of the CEA with its different centers, two dialogical pairs are considered.

Indeed, if a general view of the CEA is taken into account, the "centralized - distributed" pair can be represented as the pair "HOF specialists in the safety headquarter of CEA - HOF specialists and nonspecialists in the centers". Moreover, if a "center" view is taken into account, the "centralized - distributed" pair can be represented as the pair "HOF specialists - non-specialists".

More precisely, in a center, the local HOF network is basically organized into two levels:

- a centralized one, gathering one or two HOF specialists who work in a supply department in charge of safety analysis. This department is transverse for the research center.

- a distributed one, gathering the non-specialists who are the representatives of this HOF network in each nuclear facilities of the center.

This geographical distribution of the human resources of the network also matches with the role of the different groups.

Because of their centralized position (related to a center or to CEA) linked to the safety divisions of CEA, the HOF specialists are able to propose a uni- 
form framework for the development of the HOF approach, in particular:

- to define the methodological framework and the adapted HOF methods to apply to the safety cases,

- to apply these methods and improve them by taking the feedback of their application into account,

- to capitalize on the results of HOF studies and afterwards propose to all the members of the network the results of the HOF analysis which come from the different distributed human resources of the network.

Indeed, the HOF specialists are grouped together into a "functional entity" called "Pôle de compétences" which is in charge of these latest objectives.

Moreover, the HOF non-specialist who are located in the nuclear facilities, have a position "near the field" facilitating the identification of events or difficulties that humans can encounter in their daily life at work. They need simplified tools and methods to carry out a first level analysis of the working situations. They also need to meet the other members of the network in order to exchange information on the way to implement HOF.

So, to take into account the complementary needs of the members of the HOF network, the specialists organize two kinds of meetings that appear as conditions to enhance the framework of the HOF approach:

- meetings to share information and work practices (whole network or part of network located in one center),

- working groups to provide tools adapted to the needs of the HOF non-specialists.

Thus, this general organization of the HOF network of the CEA, by sharing the theoretical and practical approaches appears as a positive condition: firstly, to provide tools adapted to the needs of the HOF non-specialists, and secondly, to develop and to diffuse a common HOF culture.

\subsection{Microscopic-macroscopic levels of HOF methods to analyse the working situations}

HOF methods, which were developed, are based on some of the same concepts as those which are used in the nuclear safety analysis (barriers, detection, mitigation...).

To enrich the HOF specialists' studies in the CEA facilities, a specific double approach has been developed which is carried out according to two comple- mentary points of view: a "macroscopic" one and a "microscopic" one $[5,6,12]$.

The "microscopic" analysis is related to the principles of the Failure Mode and Effects Analysis view applied to human failures. It mainly examines the local failures of humans who are responsible for sensitive operations (failures of tools, machines... are not considered). The "macroscopic" analysis deals with the general provisions about the working situations which enable to ensure the general safety of the system from a HOF point of view.

Thus, thanks to the micro/macro analyses, both local and global levels are examined.

On the one hand, the microscopic point of view is focused on the humans and the functioning of the working situations. A working situation is a set of humans and technical devices that are grouped together to achieve objectives in the workplace. The microscopic point of view deals with the quality of the working situations. The objective is to understand what is the impact of the working situations on the difficulties and failures of the humans. Afterwards, ways of improving the working situations are proposed in order to avoid the human failures which occurred and could occur again in the future, to be able to detect them if they occur and to limit the consequences in such cases.

On the other hand, the macroscopic point of view is focused on the management of the working situations. The two basic purposes are the design and the maintenance of the working situations in order to avoid the deficiencies that could occur.

The feedback from several years of application in CEA facilities has successfully demonstrated the benefits of this double approach, highlighting the complementarities of the microscopic and macroscopic levels of HOF methods.

\section{Discussion: how to continuously improve these three dialogical pairs}

\subsection{The differences between the elements of a pair}

Edgar Morin provides some information about these differences [13]. Indeed, it seems to be important to keep the differences which exist between the two elements of a pair. Otherwise, the complementary aspects will disappear (with the antagonisms) since the elements are linked together. One way is to regulate the competitive aspects. For example, considering the pair specialist - non-specialist actors of the 
network, a general procedure (a kind of framework) has been developed to indicate who and what is required as the means/methods to use. Moreover, to integrate the analysis of working situations into the classical safety analysis and then contribute to enrich it, the HOF analysis of specialist has to be both near a safety analysis to be integrated inside and distant to bring worthwhile information.

\subsection{The balance between the elements of a pair}

6. It is also important to find and maintain a balance between the two elements of the pairs [14]. In this way, if an element which composes a dialogical pair is altered, an action on the other one is required in order to restore this balance. For example, it means to look for, in each center, an adequate balance between the number of specialists and non-specialists, possibly resulting in an increase of the number of HOF specialists. In this way, it might also induce some changes in the dialogical pair «centralized - distributed » (considering the general view) since the element «distributed» would also increase as a consequence. Moreover, as regards the dialogical pair: micro-macro, the levels of these two elements will be both increased since the methods used are going to be improved in the near future.

\section{Conclusion}

This communication proposed some conditions for successful integration of HOF into the nuclear safety analysis. These conditions are represented as dialogical pairs whose method of improvement is basically to increase and/or to decrease the level of each element of the pairs.

To go further, one way could be to search the limits of application of this concept of dialogical pair. Indeed, all the conditions for successful integration of $\mathrm{HOF}$ in the nuclear safety analysis are not formed in this way. By contrast, it could be also interesting to try to improve how to point out other pairs. Indeed, how to find dialogical pairs is not really precisely defined in this communication and perhaps other pairs exist and would be worthwhile to examine... Finally, a third way could be to focus the approach of dialogical pairs, not only on the HOF network but also on different other populations such as for exam- ple experimenters, facility managers, safety experts and operators.

\section{References}

[1] I. Barnabe, G. Hernandez, A. Delamotte, A. Taieb-Lutton and J.-F. Vautier, The LOFH method: a new subjective work analysis, Paper presented at the $17^{\text {th }}$ Congress of the International Ergonomics Association (IEA), Beijing, China, 2009.

[2] C. Blatter, S. Garandel, P. Gilles and J.-F Vautier, Concevoir un retour d'experience prenant en compte les facteurs humains / Designing a field experience taking Human Factors into account, Paper presented at the $17^{\text {th }}$ Lambda Mu Congress of Institut pour la Maîtrise des Risques (IMdR), La Rochelle, 2010.

[3] International Atomic Energy Agency, Safety Culture, INSAG Report 75, INSAG-4, Vienna, 1991.

[4] J.-L. Le Moigne, Edgar Morin, le génie de la Reliance, Synergies Monde 4 (2008), 177-184.

[5] C. Lipart, L'analyse des FH\&O dans les démonstrations de sûreté : en quoi la pratique fait évoluer les organisations et contribue à faire évoluer les modèles du fonctionnement de l'homme au travail, Paper presented at the $44^{\text {th }}$ Congress of the Société d'Ergonomie de Langue Française (SELF), Toulouse, France, 2009.

[6] A. Mengolini and J.-F. Vautier, How to improve safety using a micro-macro ergonomic approach, Paper presented at the $16^{\text {th }}$ Congress of the International Ergonomics Association (IEA), Maastricht, The Netherlands, 2006.

[7] E. Morin, La Méthode, La Nature de la nature (t. 1), ed., Le Seuil, 1977 , p. 80

[8] E. Morin, Introduction à la pensée complexe, ed., Du Seuil, Paris, 1990.

[9] E. Morin, Pour une réforme de la pensée. Entretiens Nathan des 25 et 26 novembre 1995, Editions Nathan, 1996.

[10] J. Reason, E. Hollnagel and J. Paries, Revisiting the Swiss cheese model of accidents, Eurocontrol EEC Note No. 13/06, 2006.

[11] M. Tosello and J.-F. Vautier, Présentation et illustration d'une démonstration de sûreté « facteurs humains », Paper presented at the $36^{\text {th }}$ congress of the Société d'Ergonomie de Langue Française (SELF), Montréal, Canada, 2001.

[12] M. Tosello, J.-F. Vautier and B. Sevestre, A new study of human factors in the nuclear safety field Paper presented at the $15^{\text {th }}$ Congress of the International Ergonomics Association (IEA), Seoul, Korea, 5, SAFETY V, 2003.

[13] N. Vallejo-Gomez, La pensée complexe : Antidote pour les pensées uniques, Entretien avec Edgar Morin, Synergies Monde 4 (2008), 249-262.

[14] J.-F. Vautier and C. Blatter, Organisation globale de la sécurité, Workshop, $15^{\text {th }}$ Lambda Mu Congress, Lille, France, 2006.

[15] J.-F. Vautier, M. Tosello, I. Barnabe, C. Lipart, F. Leveque, G. Hernandez, M. Dupont, S. Dutillieu, S. Quiblier, V. Barriere and N. Baussart, Développement du réseau Facteurs Humains et Organisationnels (FH\&O) du CEA : un témoignage réflexif, Paper presented at the $46^{\text {th }}$ congress of the Société d'Ergonomie de Langue Française (SELF), Paris, France, 2011 